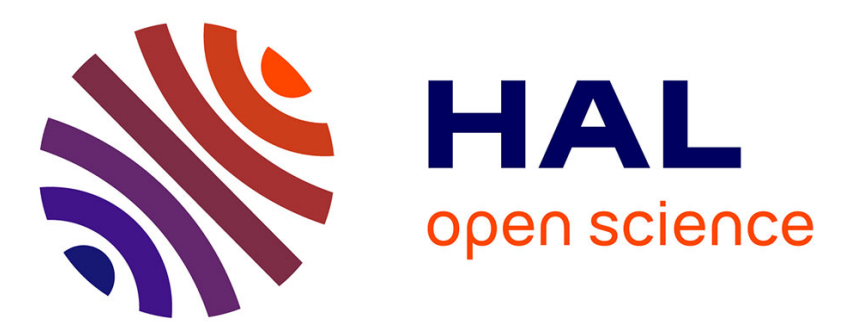

\title{
Characterization of piezoelectric materials : old and new crystals
}

B. Capelle, A. Zarka, J. Schwartzel, J. Detaint, E. Philippot, J. Denis

\section{To cite this version:}

B. Capelle, A. Zarka, J. Schwartzel, J. Detaint, E. Philippot, et al.. Characterization of piezoelectric materials: old and new crystals. Journal de Physique IV Proceedings, 1994, 04 (C2), pp.C2-123-C2134. 10.1051/jp4:1994215. jpa-00252483

\section{HAL Id: jpa-00252483 https://hal.science/jpa-00252483}

Submitted on 1 Jan 1994

HAL is a multi-disciplinary open access archive for the deposit and dissemination of scientific research documents, whether they are published or not. The documents may come from teaching and research institutions in France or abroad, or from public or private research centers.
L'archive ouverte pluridisciplinaire HAL, est destinée au dépôt et à la diffusion de documents scientifiques de niveau recherche, publiés ou non, émanant des établissements d'enseignement et de recherche français ou étrangers, des laboratoires publics ou privés. 


\title{
Characterization of piezoelectric materials: old and new crystals
}

\author{
B. CAPELLE, A. ZARKA, J. SCHWARTZEL ${ }^{*}$, J. DETAINT ${ }^{*}$, E. PHILIPPOT ${ }^{* *}$ and J.P. DENIS ${ }^{* * *}$ \\ L.M.C.P., Universités Paris VI et Paris VII, CNRS, 4 Place Jussieu, 75252 Paris cedex, France \\ ${ }^{*}$ C.N.E.T., Laboratoire de Bagneux, 196 Av. H. Ravera, 92225 Bagneux, France \\ ** L.P.C.M.S., Université de Montpellier, case 03, 34095 Montpellier, France \\ ${ }^{* * *}$ Crismatec, 2 rue des Essarts, ZI des Mayencins, 38610 Gières, France
}

\begin{abstract}
Some piezoelectric materials obtained by the hydrothermal growth method or by the Czochralsky growth method are studied. Their crystalline quality has been determined by $\mathrm{X}$-ray topography using the synchrotron radiation at LURE (Orsay, France). In the case of the gallium phosphate the growth process with berlinite seeds has been studied by the section topography method. The use of berlinite seeds seems to be a good process to obtained rapidly large good seeds of gallium phosphate and therefore large crystals.

Some results concerning the vibration modes and in some cases the interactions between the defects and the acoustic vibrations are presented.
\end{abstract}

\section{INTRODUCTION}

The piezoelectric materials are obtained by two main growth methods. The first one is the hydrothermal growth method used for quartz, berlinite and phosphate gallium(1) and the second one is a pulling method, the Czochralsky growth method,which is used, for instance, for lithium tantalate(2), lithium niobate and langasite.

The assessment of these materials perfection and in the case of the gallium phosphate the study of the growth process by X-ray topography are presented as some results concerning the vibration modes and in some cases the interactions between the defects and the acoustic vibrations.

It is possible to classify the piezoelectric materials in old materials like quartz, lithium tantalate and new ones like gallium phosphate and langasite, but they will classify by the growth method because the different types of defects depend on the corresponding growth method. 
The different materials have been studied by reflection or transmission topography conventionnal or stroboscopic(3) using the white radiation delivered by the DCI synchrotron at LURE in France.

\section{THE MATERIALS OBTAINED WITH THE PULLING METHOD}

\subsection{The lithium tantalate}

For crystals or filters too thick to be examined in a transmission Laue setting, reflection setting with an oblique incidence was used. In this case the film was adjusted parallel to the sample at a distance $(10-20 \mathrm{~cm})$ choosen to obtain in each case a good compromise between the resolution and the separation of the diffraction spots. For the thinner plates the conventional transmission Laue setting was used.

The figure 1 shows two reflection topographs of two slices of lithium tantalate. In 1-a we can observe one half of a Y-cut of a 2 inch crystal. In this topograph corresponding to a diffraction vector having a direction close to the normal to the surface, we can observe a network of dislocations and black or white contrasts due to the subgrain boundaries. In spite of the small thickness observed of the sample, the dislocations density is quite large (several hundred by square centimetre on the topograph). The contrasts corresponding to the subgrain boundaries are white lines if the relative orientations of the adjacent subgrains are such that the corresponding diffracted beams are divergent. This gives a white region without image. In the case of relative orientations giving a convergence of the diffracted beams a darker

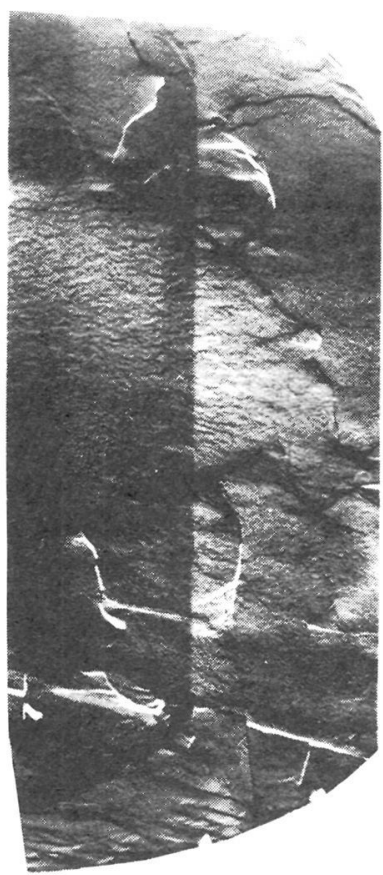

a

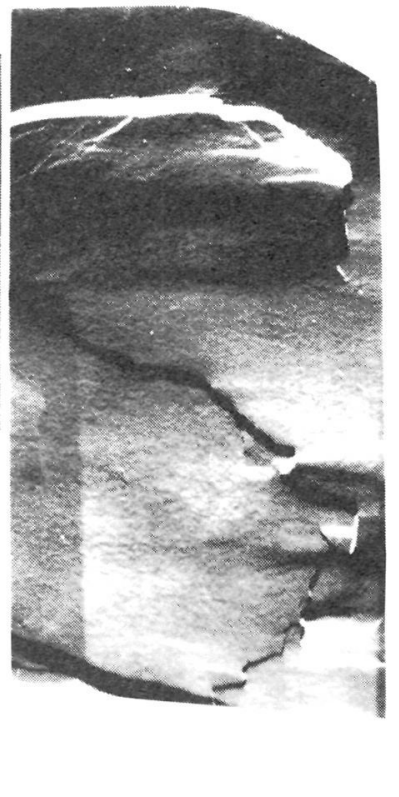

b contrast is obtained due to the overlapping of the images. It is possible, from the exact knowledge of the experimental setting, and the measurements of the width of the black or white lines, to calculate the relative orientations of different subgrains. The examined part of this plate, about $3.7 \times 1.7 \mathrm{~cm}^{2}$, contains nearly ten subgrains, the larger having an area exceeding two square centimetre.

In fig $1-\mathrm{b}$ is presented a topograph of a $\mathrm{Y}-45^{\circ}$ slice. About 4 subgrains can be observed in this topograph and the sample presents the same dislocations density that the precedent one. 
Two transmission topographs are represented in the figure 2 , the area of each is about $0.8 \times 1.6 \mathrm{~cm}^{2}$. The thickness of these Y-45 lithium tantalate slices is 100 microns.

The topograph in fig 2-a shows an interesting feature of the dislocation network. These dislocations converge, probably to form subgrain boundaries. It seems that two families of dislocations appear: the first one corresponds to dislocations which give images roughly directed in the pulling direction and the second to dislocations which are organised in convergent network situated in a plane closer to that of the plate. It is probable Fig.2-a and b: transmission topographs of thin that this second family has an

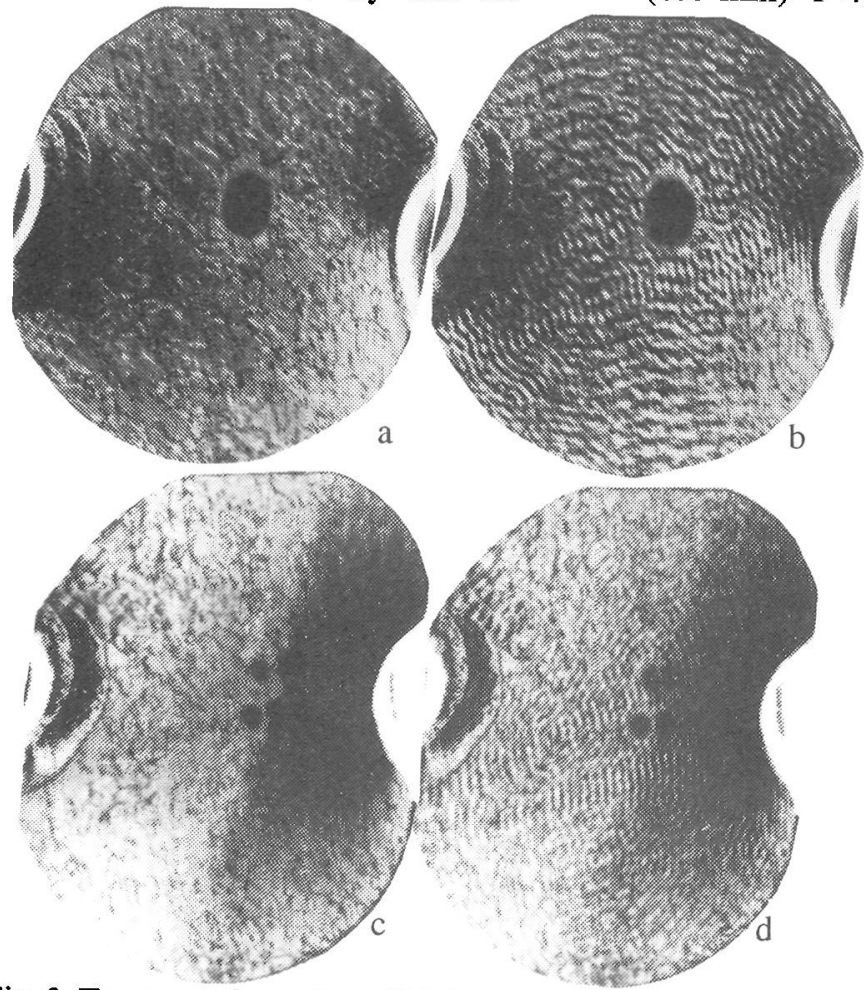

Fig.3-Topographs of a lithium tantalate resonator.

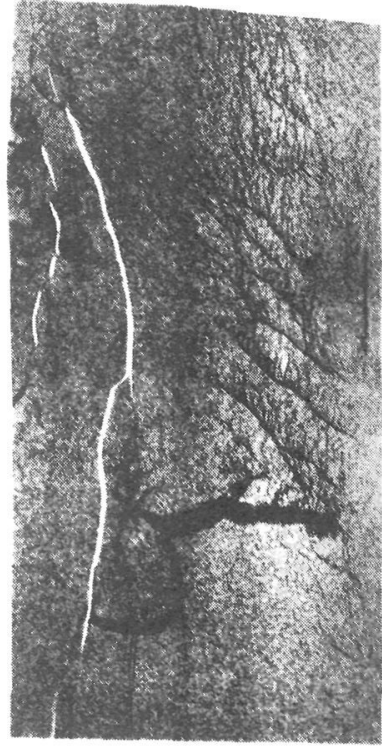

a

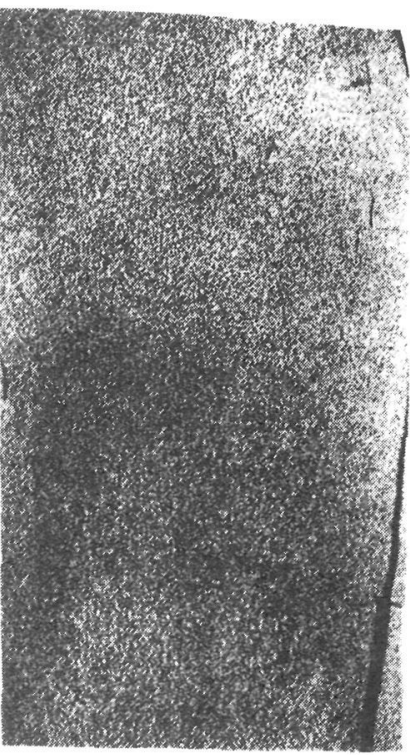

b origin which is different from the first one. This fact, which is important to understand the mechanism of apparition of the subgrains and also to optimize the growth and cooling conditions of the crystals, needs further investigations

On the topograph, in fig 2-b, a quite large density of dislocations homogeneously distributed across the surface and only one subgrain boundary can be observed.

On the whole, the crystal quality of the lithium tantalate seems to be sufficiently good to make bulk and wave devices, owing to the large dimensions of the observed subgrains.

An example of topographs of a lithium tantalate resonator having small 
rectangular electrodes elongated in the $x_{3}$ direction is shown in figure 3 . This resonator was examined using stroboscopic and conventional topography techniques; in fig $3-\mathrm{a}$ the $\mathrm{u}_{1}$ component of the fundamental fast shear mode observed in conventional conditions and in stroboscopic conditions in fig 3-b. In 3-c and 3-d we have the same observation but obtained for the $u_{3}$ component. From these figures it can be concluded that the trapped fast shear mode is coupled to a progressive component that extends over the total surface of the plate. This progressive component appears like fringes on the topographs. With the stroboscopic setting and a slow variation of the phase shift between the resonator excitation and the synchrotron current, it is possible to observe, with an X-ray-sensitive video camera, that the non-stationary component is progressing towards the mounting clips. Thus, this progressive component, which has a large standing-wave ratio, carries a fraction of the energy outside the resonator. This fact was observed in all the examined resonators and is most probably responsible for the rather low $Q$ factor of the fundamental fast shear mode.

The mode shape of the $u_{1}$ component is elongated in the $x_{1}$ direction whereas the electrodes are elongated in the $\mathrm{x}_{3}$ direction; this indicates that the $\mathrm{X}$ cut of lithium tantalate has a large in-plane anisotropy.

\subsection{The lithium niobate}

Another material obtained by the Czochralsky growth method at a very high temperature $\left(1260^{\circ} \mathrm{C}\right)$ is the lithium niobate. Among several piezoelectric materials which can be used for S.A.W. devices, the lithium niobate has the larger coupling coefficients and hence permits to obtain filters with the largest bandwidths and other signal processing devices with a great efficiency.

The use of synchrotron radiation permits investigations of the quality of this material by X-ray topography. Such research is extremely difficult (or even impossible) with conventional sources, due to the very high $\mathrm{X}$-ray absorption of these crystals.

The figure 4 presents a reflection topograph of an $\mathrm{Y}$ $45^{\circ}$ slice. This topograph is obtained with a diffraction vector having direction close to the normal to the surface. It may be observed a network of dislocations and a black and white contrast due to a subgrain boundary. The quality of this crystal is good enough.

An X-ray topograph of

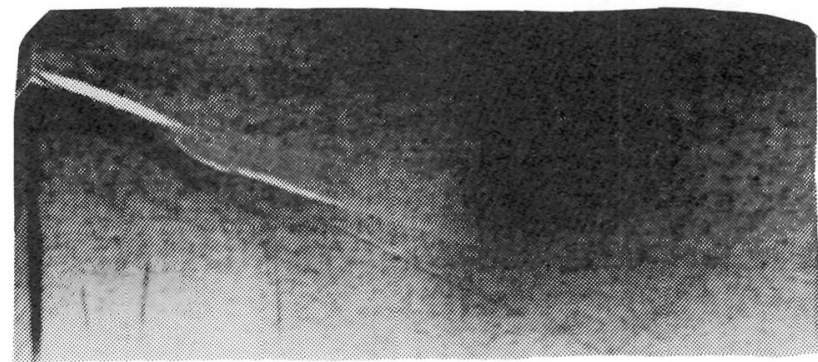

Fig.4-Reflexion topograph of an $\mathrm{Y}-45^{\circ}$ slice. an $\mathrm{X}$-cut slice from a crystal grown in the $\mathrm{Y}$ direction is shown in the figure 5 . This topograph was obtained with the reflection setting and it may be observed that the quality of this crystal is good, very few dislocations are present and it does not present subgrains.

When the crystal is thin enough it is possible to study it with the transmission Laue setting. The figure 6 shows an example of a slice studied by 


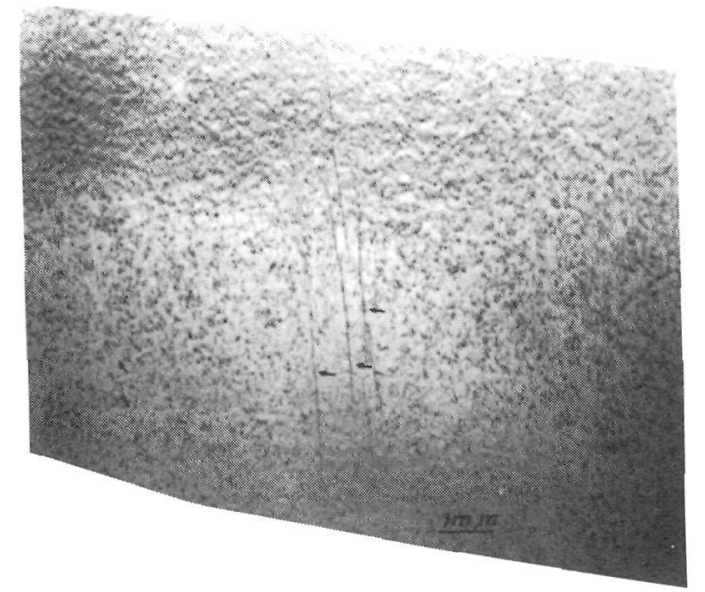

Fig.5-Transmission topograph of an $\mathrm{X}$ cut slice.

transmission Laue setting. It corresponds to a BAW resonator which is an $\mathrm{Y}+36^{\circ}$ cut plate of about $100 \mu \mathrm{m}$ thick. Two types of defects are visible: a subgrain boundary and dislocations. In fig 6-b the plate is vibrating in an overtone thickness stretch mode (bulk waves). The central dark part corresponds to the

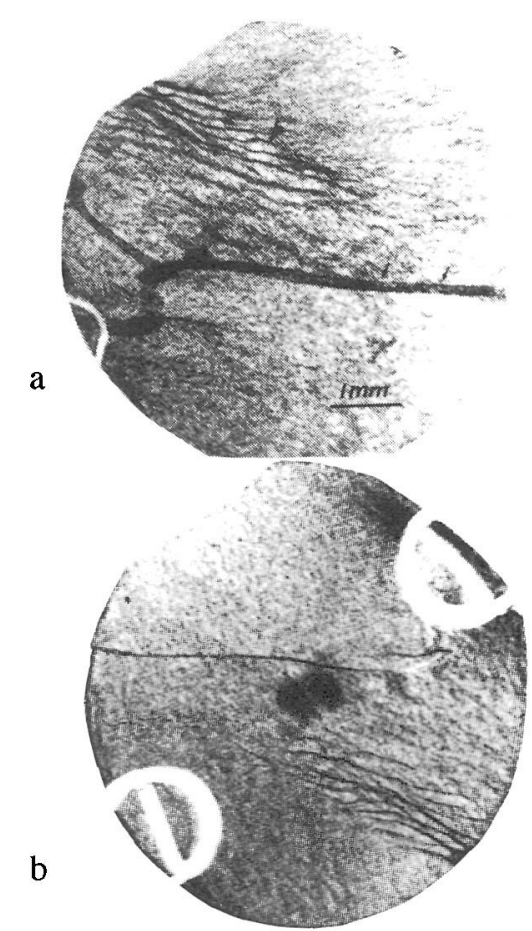

Fig.6-Topographs of a BAW resonator without vibration in $a$ and with vibration in $b$.

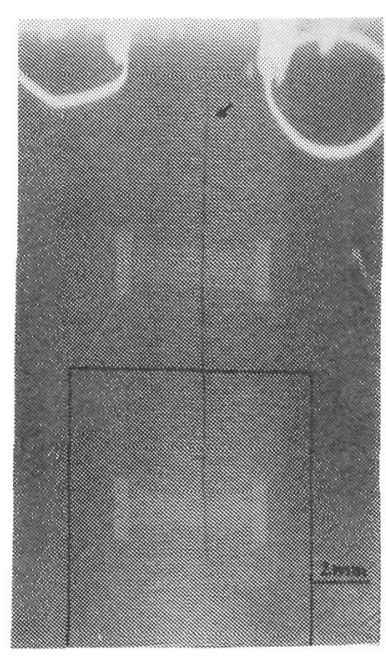

a

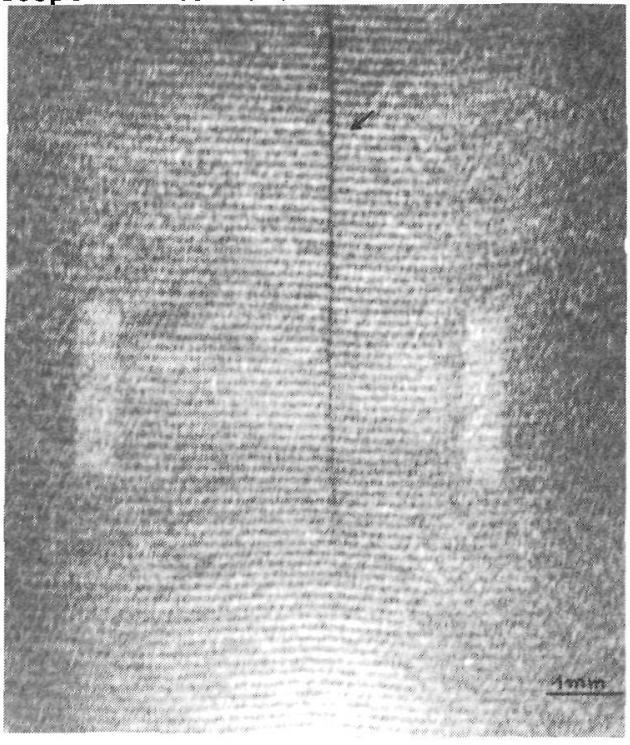

b

vibrating zone. The subgrain boundary presents another configuration because the corresponding diffraction vector is different than the previous one in 6 -a.

The lithium niobate, with its physical properties, can be used also for S.A.W. devices with a great efficiency. The figure 7 presents a stroboscopic topograph of the waves generated by an unapodized transducer. The wave fronts are

Fig.7-Stroboscopic topograph of an un-apodized transducer with a subgrain in the middle.

straight lines and the waves have constant 
amplitude in the transversal direction except at the extremities of the aperture. In this plate there is a subgrain boundary, in the middle, perpendicular to the wave fronts, which induces a phase shift between the waves which propagate on the two sides of the boundary. In the enlargement in fig 7-b, one can see better the phase shift. This observation shows the importance of the role of a subgrain boundarie, even with a weak disorientation, on the wave propagation.
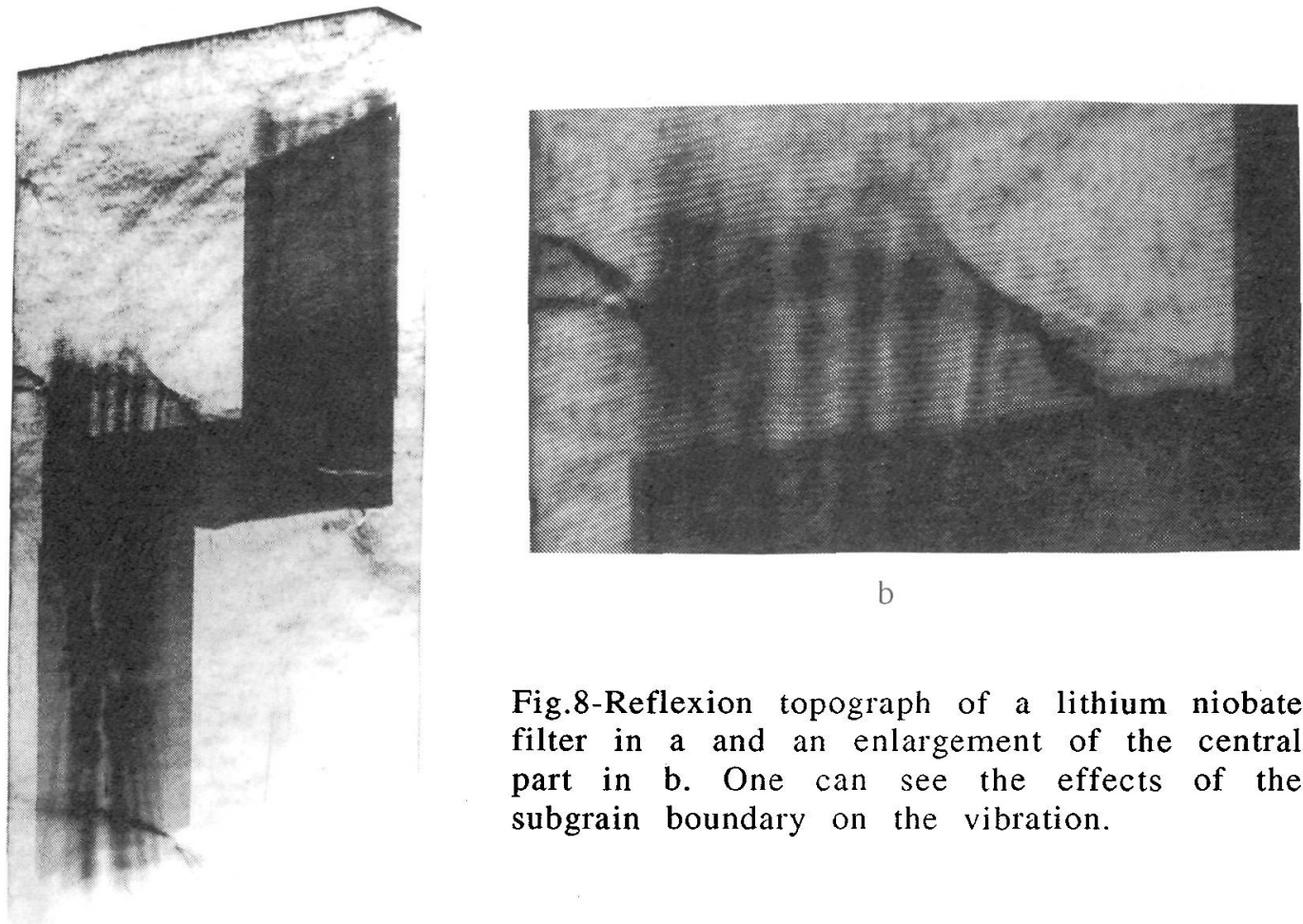

b

Fig.8-Reflexion topograph of a lithium niobate filter in $a$ and an enlargement of the central part in b. One can see the effects of the subgrain boundary on the vibration.

In the figure 8 is presented a topograph of a filter. This topograph obtained with the reflection Laue setting shows two subgrains boundaries and some parallel contrasts on the top of the sample due to a scratches lattice made in the back side of the sample. In the central part, an acoustic absorbent has be deposited. On the topograph on the figure 8-a the filter is vibrating and one can see the effects of the subgrain boundary on the vibration. The zone in the middle with a subgrain boundary is enlarged on the figure $8-b$. One can see that the surface acoustic wave presents phase shifts due to the subgrain boundary but its principal effect is that it deforms the end of the acoustic wave.

In spite of the deffects observed in this material, this study shows that the crystal quality of the lithium niobate has been greatly enhanced in the recent years in comparison with those produced some time ago and that it is a good material for SAW devices.

\subsection{The Iangasite}

The last piezoelectric material obtained with a pulling growth technique studied in this paper is the langasite. 


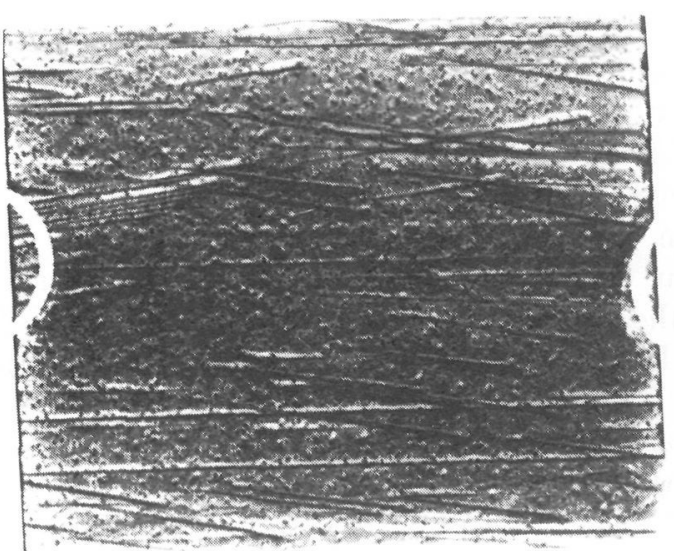

a

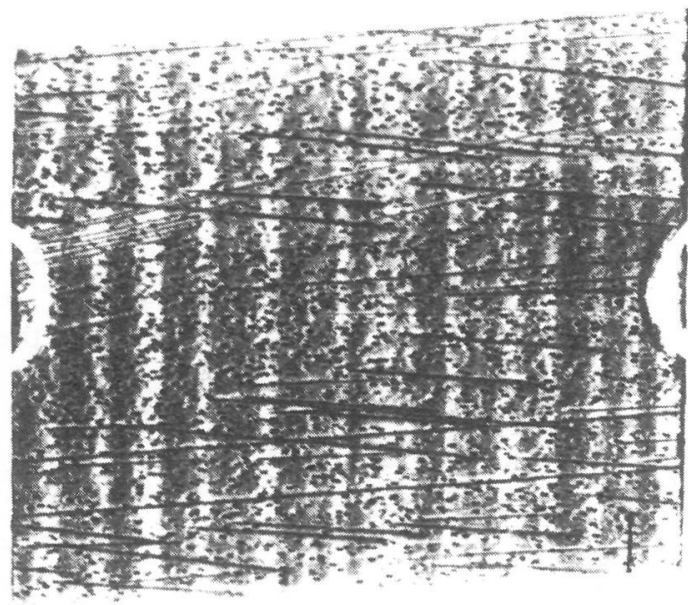

b

Fig.9-Transmission topographs of a langasite crystal obtained simultaneously with two different diffraction vectors.

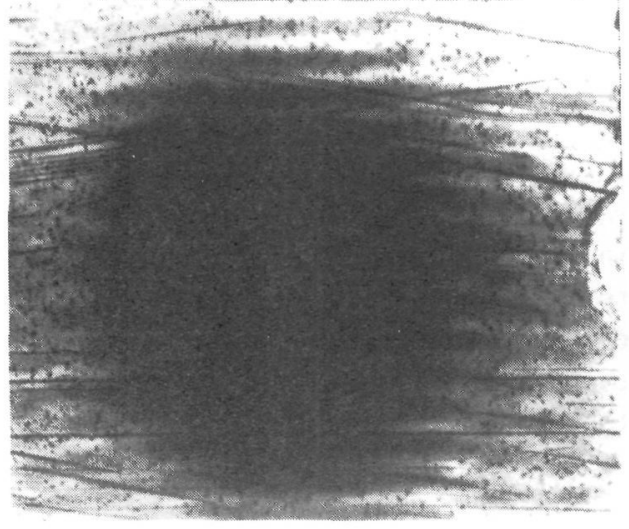

a

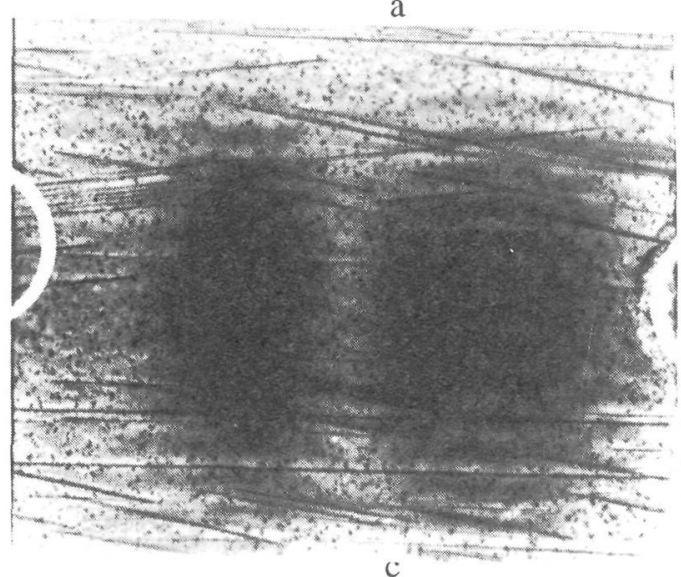

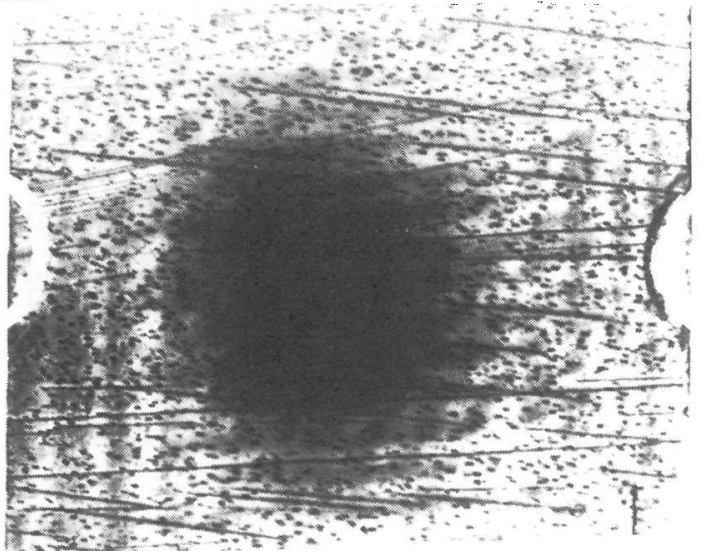

b

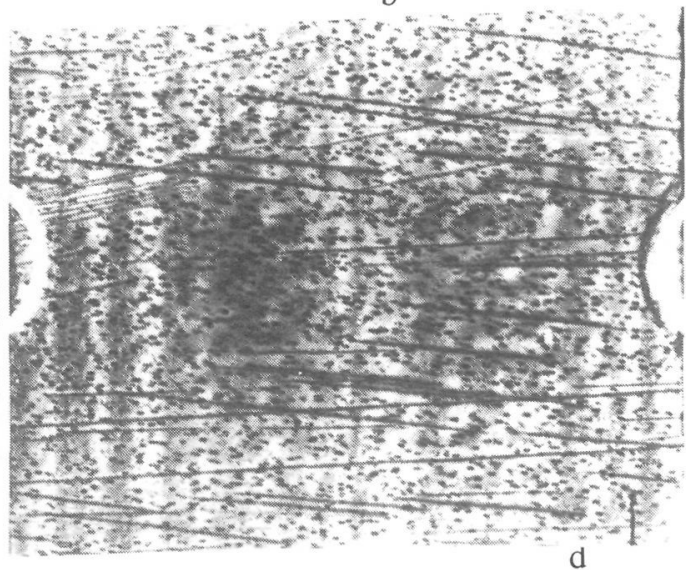

Fig.10-Transmission topographs of the same resonator that in the figure 9 but with the fundamental mode ( $a$ and $b$ ) and the first antisymetrical mode ( $c$ and $d$ ). 
The two topographs, in the figure 9, obtained simultaneously with two different diffraction vectors show a langasite crystal. The dislocations which appear are parallel with the pulling axis and their density is quite similar to the density of the dislocations in the $Z$ zone of a good quartz crystal. The little dots correspond to the images of precipitates. The large bands alternatively black and white which appear on the bottom are growth bands. They are perpendicular to the pulling axis and do not appear on the previous topograph because the diffraction vector is perpendicular to their displacement vector which is parallel to the pulling axis. Indeed, generally the displacement vector of the growth bands is perpendicular to the bands. The figure 10 shows topographs of the same crystal. The topographs of the same column are obtained with the same diffraction vector and the topographs of the same line are obtained simultaneously with two different diffraction vectors. In fig $10-a$ and $10-b$ the fundamental mode and in $10-\mathrm{c}$ and $10-\mathrm{d}$ the first antisymetrical mode. These two modes are strongly coupled with flexure. The fundamental mode is not well trapped because the use of a too large electrode associated with a too thick crystal.

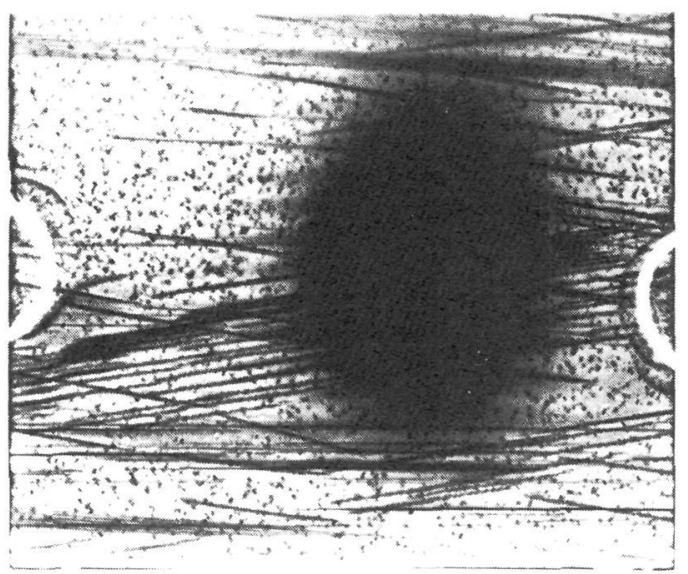

Fig.11-Transmission topograph of the fundamental vibrating mode of a langasite resonator.

In the figure 11 is presented another example of langasite crystal with the fundamental vibrating mode. This topograph is obtained with the diffraction vector which do not shows the growth bands. We can see the same density of dislocations that in the previous sample but with an inhomogeneous distribution. In this topograph the vibrating mode is diffuse because the numerous parallel dislocations guide the energy like in the quartz resonators.

This new material has already a crystalline quality similar to that of the quartz and with the used growth technique it is possible to obtain large crystals without growth sectors, all the material can be used.

\section{THE MATERIALS OBTAINED WITH THE HYDROTHERMAL GROWT] METHOD}

\subsection{The berlinite}

The figure 12 shows topographs of an AT-cut berlinite crystal. In the topograph of the crystal without vibration (Fig.12a) some vertical dislocations appear and stop at the limit of the seed on the bottom of the resonator. The different vibration modes visible on the others topographs in fig 12-b, c, d and e are the fundamental mode, the third overtone, the fifth overtone and the seventh overtone respectively. The fundamental mode is not well trapped and presents flexure. The overtones are well trapped but flexure components are very 
important in particular with the third overtone. All these modes are elongated in the $X$ direction.

One important problem for the berlinite is the too small size of the crystals. But it is difficult to have large seeds which are necessary to obtain large crystals because $\mathrm{V}_{\mathrm{y}}$ is equal to zero. Also, one solution is the pasting of few little seeds with a very high precision for the relative orientation between the different parts to obtain a large seed. To make it, it is necessary to find a paste which resists at the growth solution. A transmission topograph of a good crystal obtained with this pasted seeds method is shown in the figure 13. One can see the emplacement of the pasting marked by dislocations which appear because there is always a small defect of orientation between the different initial seeds. But it is possible to decrease the number of the dislocations with the crossed growth method. The fringes which are visible on this topograph are due to a slow variation of the crystal parameters which induces an $X$-ray diffraction effect. The pasting of the seeds, seems to be a good solution to obtain berlinite large seeds and then large crystals.

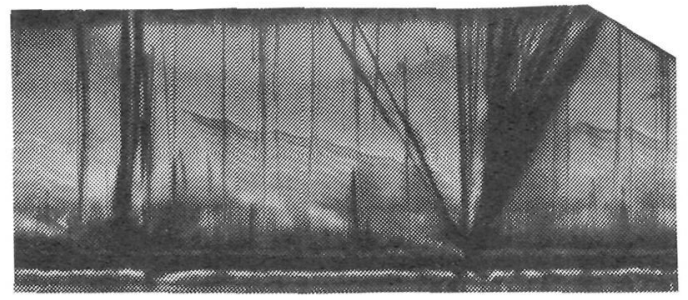

Fig.13-Topograph of a berlinite crystal obtained with the pasted growth method
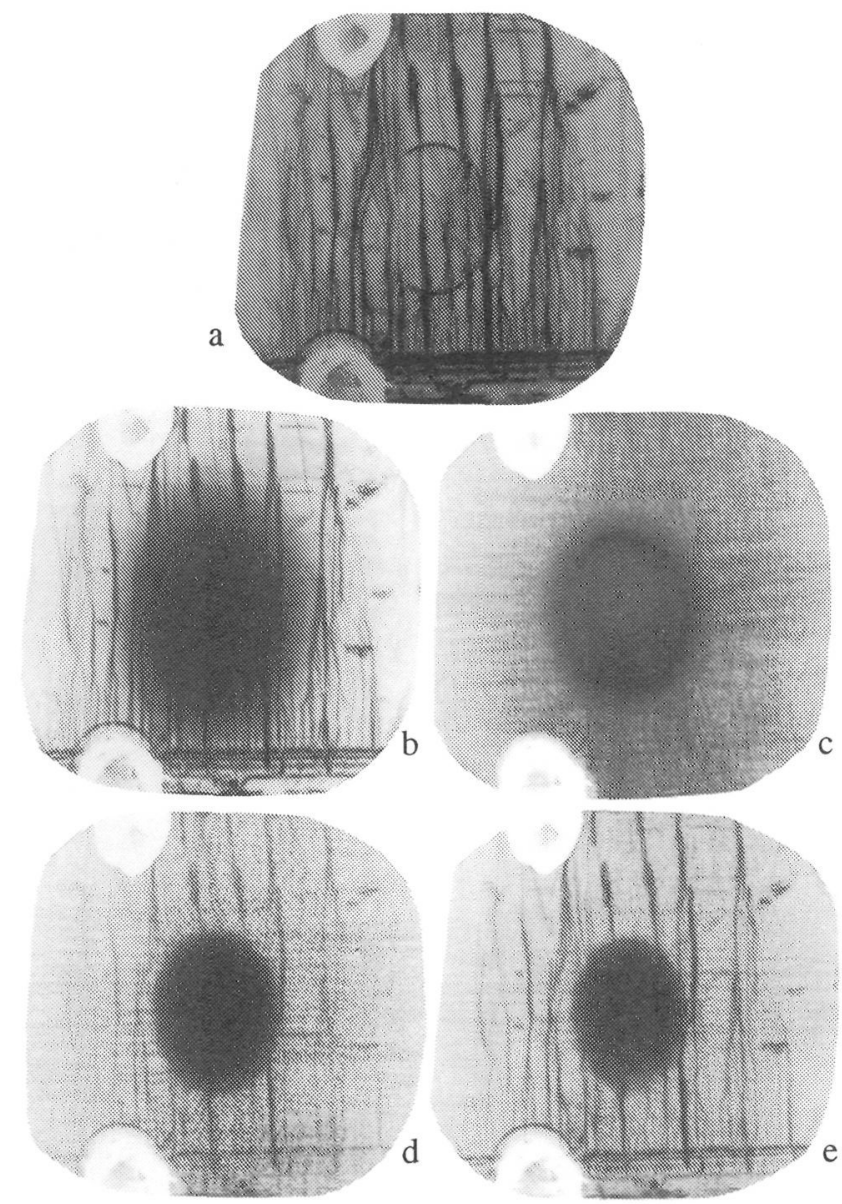

Fig 12-Topographs of an AT-cut berlinite resonator without vibration in $a$ and with vibration in $b$, $c, d$ and $e$.
As the berlinite, an important problem for the gallium phosphate is the seed and in particular the size of the seed. A fine solution, used by the group of Etienne Philippot at Montpellier, consists of the use of a berlinite seed for the growth of the gallium phosphate. Indeed, large berlinite seeds (four to eight centimeters 
long) are now available.

We become by a growth with a $Z$ berlinite seed in sulfuric acid and vertical thermal gradient (VTG) method $(4,5) . V_{X}$ being the only significant growth rate, flat crystals are obtained with a very thin epitaxy of gallium phosphate in the $\mathrm{Z}$ direction. We continue the growth from this crystal in phosphoric acid and composite gradient method to have a significant $V_{Z}$ growth rate. This method, with nutrient in the colder side of the glass autoclaves, allows to obtain larger crystals than the slow heating (SHT) method. The fluid circulation, from cold to hot zone due to the horizontal temperature gradient, is done at constant temperature or coupled to a slow heating rate of 0.25 to $1.0^{\circ} \mathrm{C} /$ day. In both cases, quite good results are obtained for the crystal growth experiments.

Then, we can cut gallium phosphate $X$ seeds for a growth in sulfuric acid and the VTG method or $\mathrm{Z}$ seeds for a growth in phosphoric acid and the SHT method or the composite gradient method.

Two traverse topographs of the crystal shown in the figure 14-b are in the figures 14-a and 14-c : the X zone and the top part of the berlinite seed (14-a) and the berlinite seed and a part of the $\bar{X}$ zone (14-c) appear. We can see, at the interface between the berlinite seed and the gallium phosphate $X$ zone, strains which decrease when the distance to the seed increases. To analyse the epitaxial growth of the gallium phosphate on the berlinite seed the X-ray section topography is a very good method. The results are shown in the figure 15: in 15-e the same traverse topograph that in the figure $14-\mathrm{a}$ and in $15-\mathrm{a}, \mathrm{b}, \mathrm{c}$ and $\mathrm{d}$ different section topographs obtained with a fine horizontal slit.

In fig 15-c and $d$ two section topographs made at the seed-level. We have here the case where the crystal is made with 3 parts, a berlinite substrate, the seed, and two epitaxial gallium phosphate growth on both sides. These two section topographs are obtained with two different diffraction vectors. Due to the strains, separated images for the different parts are obtained. In fig 15-d we can see, at the bottom, the image of the berlinite seed and above the two images of the epitaxial gallium phosphate. The aspect of these images is due to the different values of the strain at each point of the interface. In fig 15-c we have 
a

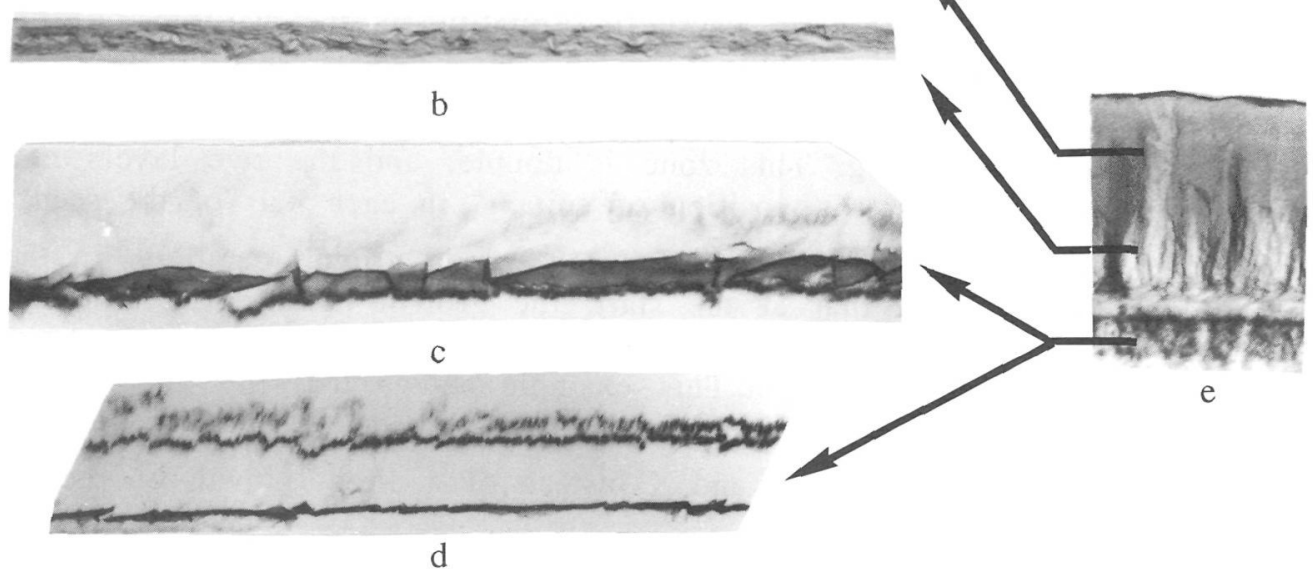

Fig.15-In a, b, c and d section topographs made above the seed ( $a$ and $b$ ) and at the seed-level ( $c$ and $d$ ). In e traverse topograph of the $X$ zone and the top part of the berlinite seed

the same thing, but the seed image is like a ribbon. The two other section topographs show that the quality increase far from the seed. In fig 15-b we have

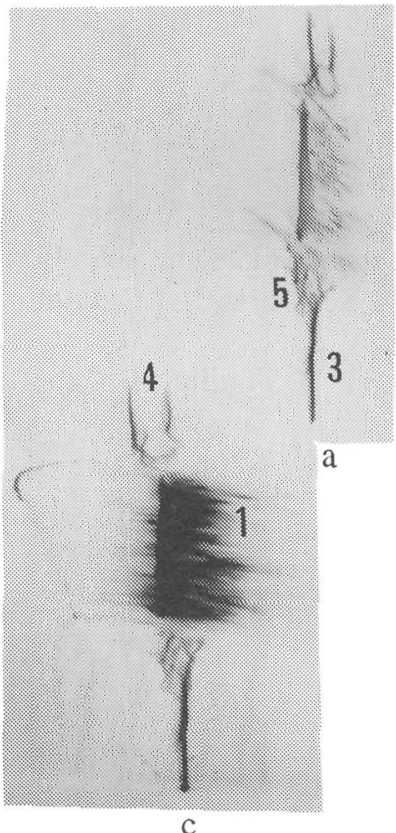

Fig.16-Section topographs obtained with a fine vertical slit. many defects and in 15-a the quality become better

If we put a fine vertical slit we obtain one another set of section topographs (Fig.16). These three section topographs of another crystal have been obtained simultaneously on the same Laue pattern but with different diffraction vectors. In each of these topographs three zones are visible. The central zone is composed of the seed image of the berlinite which appears as a well defined line and the images of the layers of the gallium phosphate which are very perturbed. The two layers show images which are very different at the level of the seed. The layer at the right, in 1, appears like a large contrast in an irregular strip. On the contrary, the layer in the left do not show any image as in Fig16-a and 16-b, or a very fine image which extends far from the seed as in the figure16-c. 
The two other zones below and above the previous one correspond respectively to the $\mathrm{X}$ and $\overline{\mathrm{X}}$ zones. The zone $\mathrm{X}$, located in 3 on the three topographs $(\mathrm{a}, \mathrm{b}, \mathrm{c})$ is well defined and shows a good crystalline quality of the gallium phosphate. However the interface between this zone and the seed is very disturbed. The quality of the crystal becomes progressively better far from the seed. The $\overline{\mathrm{X}}$ zone, in 4, seems very interesting. This zone is double, and the two layers of the gallium phosphate which are in position of epitaxy, in each part of the seed, are separated in the $\bar{X}$ zone and give two crystals which growth parallely. In the $X$ zone, it may be observed that at the start, the growth had a double direction. This is visible in 5 on the figure 16-a. Progressivelly, the two parts joint together and give rise to a single $X$ zone.This example shows that the growth of the gallium phosphate is obtained from the epitaxial layers on the berlinite seed which develop to give the zones $\mathrm{X}$ and $\bar{X}$ instead of a direct growth on the lower and the upper parts of the seed.

This result is interesting and shows how to obtain large good seeds of gallium phosphate.

\section{Conclusion}

With all these examples one can say, first, that the translation and section topography conventionnal or stroboscopic is a very good method to characterize the piezoelectric materials and to analyse the vibration modes, secondly, that the new materials like the langasite and the gallium phosphate are very promising, their crystalline quality being already very good.

\section{References:}

(1) E.Philippot, A.Ibanez, A.Goiffon, B.Capelle, A.Zarka, J.Schwartzel, J.Detaint, Proc. of the 6th European Frequency and Time Forum, Noordwijk (1992), p.383-388.

(2) A.A.Ballman, J.Am.Ceram.Soc., 48(1965), 112-113.

(3) A.Zarka, B.Capelle, J.Detaint, J.Schwartzel, J.Appl.Cryst., 21(1988), 967-971.

(4) J.C.Jumas, A.Goiffon, B.Capelle, A.Zarka, J.C.Doukhan, J.Schwartzel, J.Detaint, E.Philippot, J. of Cryst. Growth, 80(1987), 133.

(5) E.Philippot, A.Goiffon, M.Maurin, J.Détaint, J.Schwartzel, Y.Toudic, B.Capelle, A.Zarka, J. of Cryst. Growth, 80(1990), 713-726. 